\title{
Influence of Modulation Rate on Energy Efficiency for Relay-aided Transmission: Decode-and-forward Protocol
}

\author{
SUN Rui ${ }^{1, a}$, WANG Tao ${ }^{2, b}$,Chen FangFang ${ }^{3, c}$, HUANG QingHua ${ }^{4, d}$ \\ ${ }^{1,2,3}$ Key Laboratory of Specialty Fiber Optics and Optical Access Networks, \\ School of Communication \& Information Engineering, \\ Shanghai University, Shanghai 200072, P. R. China \\ aemail: 13720880@shu.edu.cn, bemail: twang@shu.edu.cn, \\ cemail: cffshdx@163.com, email: qinghua@shu.edu.cn
}

\begin{abstract}
Keywords: Energy Efficiency; Decode-and-forward; Relaying Systems; Modulation Rate
Abstract. In this paper, we investigate the effect of modulation rate on the energy efficiency of a two-hop communication system with the assistance of a half-duplex decode-and-forward (DF) relay, considering the given bit error rate (BER) and using the M-QAM modulation. First, we formulate the optimum model of transmission power, subject to the given BER. Then the minimum transmission power under the constraint of the given BER is obtained. For energy efficiency problem, the circuit power consumption of devices can not be ignored, so with the circuit power consumption considered, we derive and analyze the expression of energy efficiency based on the modulation rate. According to convex optimization theory, energy efficiency is a quasi-concave function of modulation rate has been proved. We find that when the modulation rate increases, the energy efficiency will be first increases and then decreases. Finally, the theoretical analysis is corroborated by simulation results. Moreover, it is shown that the location of relay node has effect on system energy efficiency.
\end{abstract}

\section{Introduction}

With the rapid development of wireless communication technology, the communication network and internet has evolved day by day, and the network scale expands constantly. Reducing the energy consumption of the communication industry has attracted much attention from both industry and academia.Therefore, energy efficiency, as a new kind of system performance, is becoming more and more important in mobile communication devices powered by batteries, e.g., wireless sensor networks [1]. Relaying has been widely studied as one of key strategies for improving the energy efficiency of wireless communication systems, because it can reduce the signal transmission power by breaking a long distance transmission into several short ones [2,3].

Decode-and-forward (DF) is one of the major relaying protocols under investigation. In [4], the EE for the DF-TWRT (two-way relay transmission) under relay power constraint is analyzed. Through the power allocation scheme, the total transmit power subject to a target BER is minimized at the destination for both AF and DF relaying systems [5]. Previous works are more concerned about the EE performance of one-way or two-way relay under AF/DF protocols. However, the effect of some system parameters such as modulation rate on the energy efficiency has not been researched in the previous work.

In this paper, we analyze the effect of modulation rate on the energy efficiency of a triplet communication system with the help of a half-duplex decode-and-forward (DF) relay, under the given bit error rate (BER) constraint. In contrast to precious work, the circuit power consumption is modeled as a function of the modulation rate, rather than as a constant. The expression of energy efficiency based on the modulation rate is derived and analyzed and we find after analysis that when the modulation rate increases, the energy efficiency will be first increases and then decreases. Finally, the theoretical analysis is corroborated by simulation results.

The remainder of the paper is organized as follows. The system model is introduced in Section II. In Section III, we derive the minimum transmission power under the given BER, and the EE optimization problem using modulation rate as the optimization variable is constructed. In Section 
IV, we analyze the effect of modulation rate on the energy efficiency. The simulation results are shown in this part as well. We conclude this paper in the Section V.

\section{System Model}

We consider a triplet communication link, where the half-duplex DF relay node $\mathrm{R}$ supports the communication from source $\mathrm{S}$ to destination $\mathrm{D}$, as shown in fig. 1. Data transmission is carried out in two consecutive time slots. In the first slot, $\mathrm{S}$ transmits symbols to $\mathrm{R}$, and $\mathrm{R}$ transmits to $\mathrm{D}$ in the second.

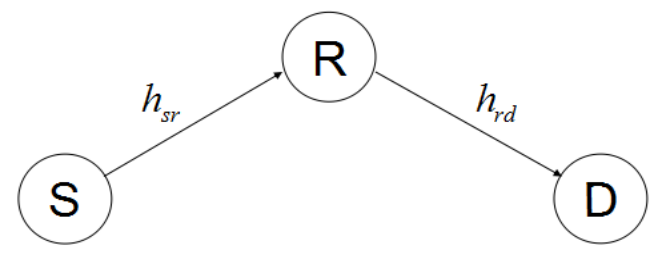

Fig. 1. A simplified three nodes model

The channel coefficient between the source and the relay and that between the relay and the destination are denoted as $\mathrm{h}_{\mathrm{sr}}$ and $\mathrm{h}_{\mathrm{rd}}$, and the channels are assumed to be reciprocal and flat-fading with bandwidth B Hz.

The receive signals at $\mathrm{R}$ and $\mathrm{D}$ are given by

$$
\begin{aligned}
& y_{s r}=\sqrt{P_{S}} h_{s r} x_{s}+n_{s r}, \\
& y_{r d}=\sqrt{P_{R}} h_{r d} x_{r}+n_{r d} .
\end{aligned}
$$

Where $x_{s}$ and $x_{r}$ represent the transmission signals at $\mathrm{S}$ and $\mathrm{R}$, moreover, $E\left[\left|x_{s}\right|^{2}\right]=1, E\left[\left|x_{r}\right|^{2}\right]=1 . P_{S}$ and $P_{R}$ denotes the power used as transmitting $x_{s}$ and $x_{r} . n_{s r}$ and $n_{r d}$ are denoted as the additive Gaussian noise terms on the two links (SR and RD) with variance $\sigma_{n}^{2}$. We can define the corresponding SNRs as

$$
\begin{aligned}
& \gamma_{s r}=\frac{P_{S}\left|h_{s r}\right|^{2}}{\sigma_{n}^{2}}, \\
& \gamma_{r d}=\frac{P_{R}\left|h_{r d}\right|^{2}}{\sigma_{n}^{2}} .
\end{aligned}
$$

For figure 1, the BERs introduced by both $\mathrm{SR}$ and RD link, denote as $\mathrm{P}_{b}^{S R}$ and $\mathrm{P}_{b}^{R D}$, thus, the whole BER $\mathrm{P}_{b}^{D F}$ can be approximately expressed as[5]

$$
\begin{aligned}
\mathrm{P}_{b}^{D F} & =\mathrm{P}_{b}^{S R}\left(1-\mathrm{P}_{b}^{R D}\right)+\mathrm{P}_{b}^{R D}\left(1-\mathrm{P}_{b}^{S R}\right) \\
& =\mathrm{P}_{b}^{S R}+\mathrm{P}_{b}^{R D}-2 \mathrm{P}_{b}^{S R} \mathrm{P}_{b}^{R D} \\
& \approx \mathrm{P}_{b}^{S R}+\mathrm{P}_{b}^{R D} \quad\left(2 \mathrm{P}_{b}^{S R} \mathrm{P}_{b}^{R D}<<\mathrm{P}_{b}^{S R}+\mathrm{P}_{b}^{R D}\right)
\end{aligned}
$$

The BER for M-QAM transmission with receive SNR $\gamma$ for AWGN channels can be approximated as the following equation:

$$
\mathrm{P}_{b} \approx \frac{2}{\theta}\left(1-\frac{1}{\sqrt{2^{\theta}}}\right) \cdot \operatorname{erfc}\left(\sqrt{\frac{a_{2} \gamma}{2^{a_{3} \theta}-a_{4}}}\right),
$$

According to [6], (5) can be further approximated to the following equation:

$$
\mathrm{P}_{b}=a_{1} \exp \left(\frac{-a_{2} \gamma}{2^{a_{3} \theta}-a_{4}}\right) \text {. }
$$

Where $a_{1}=0.2, a_{2}=1.6, a_{3}=a_{4}=1 . \theta$ is the modulation rate that is the number of bits carried by one symbol. The BERs for corresponding channels are given by (3), 


$$
\begin{array}{r}
\mathrm{P}_{b}^{S R}=a_{2} \exp \left(\frac{-a_{2} \frac{P_{S}\left|h_{s r}\right|^{2}}{\sigma_{n}^{2}}}{2^{a_{3} \theta}-a_{4}}\right), \\
\mathrm{P}_{b}^{R D}=a_{1} \exp \left(\frac{-a_{2} \frac{P_{R}\left|h_{r d}\right|^{2}}{\sigma_{n}^{2}}}{2^{a_{3} \theta}-a_{4}}\right) .
\end{array}
$$

From (7), we can obtain the transmission power in source node and relay node, denote as $P_{S}$ and $P_{R}$

$$
\begin{aligned}
& P_{S}=\frac{\left(a_{4}-2^{a_{3} \theta}\right) \sigma_{n}^{2}}{a_{2}\left|h_{s r}\right|^{2}} \cdot \ln \left(\frac{\mathrm{P}_{b}^{S R}}{a_{1}}\right), \\
& P_{R}=\frac{\left(a_{4}-2^{a_{3} \theta}\right) \sigma_{n}^{2}}{a_{2}\left|h_{r d}\right|^{2}} \cdot \ln \left(\frac{\mathrm{P}_{b}^{R D}}{a_{1}}\right) .
\end{aligned}
$$

\section{Optimum Problem Formulation}

We define the energy efficiency as the sum of transmission data bits per unit of energy, then the EE $\eta_{E E}$ is given by [4]

$$
\eta_{E E}=\frac{R_{t o t}}{P_{t o t}}=\frac{\theta B}{P_{T} / \xi+P_{C}} \text {. }
$$

Where $P_{T}=P_{S}+P_{R}$ is the total power used in transmitting data bits, $\xi$ is the efficiency of the power amplifiers for nodes $\mathrm{S}$ and the relay R. $P_{C}$ is the circuit power modeled as $P_{C}=\alpha+\beta \theta B$, i.e., with a linear rate dependence [7].

In order to obtain the maximized $\mathrm{EE}$, we first find the minimum transmission power subject to the given BER. The power optimization problem can be formulated as

$$
\begin{array}{ll}
\min & P_{T} \\
\text { s.t. } & \mathrm{P}_{b}^{S R}+\mathrm{P}_{b}^{R D} \leq \mathrm{P}_{b}^{t a r} \\
& P_{S}, P_{R}>0 .
\end{array}
$$

The optimum solution of the above problem must satisfy KKT conditions, according to this, we can deduce the BER for the link S-R and R-D, of which transmission power is optimum.

$$
\mathrm{P}_{b}^{S R}=\frac{\left|h_{s r}\right|^{2} \mathrm{P}_{b}^{t a r}}{\left|h_{s r}\right|^{2}+\left|h_{r d}\right|^{2}} \cdot \mathrm{P}_{b}^{R D}=\frac{\left|h_{r d}\right|^{2} \mathrm{P}_{b}^{t a r}}{\left|h_{s r}\right|^{2}+\left|h_{r d}\right|^{2}}
$$

The specific derivation peocess can be found in appendix A. Then we can get the minimum $P_{T}$, which satisfy the given BER.

$$
P_{T, \text { min }}=\frac{\left(a_{4}-2^{a_{3} \theta}\right) \sigma_{n}^{2}}{a_{2}} \cdot\left[\frac{\ln \left(\frac{\left|h_{s r}\right|^{2} \mathrm{P}_{b}^{t a r}}{a_{1}\left(\left|h_{s r}\right|^{2}+\left|h_{r d}\right|^{2}\right)}\right)}{\left|h_{s r}\right|^{2}}+\frac{\left.\ln \left(\frac{\left|h_{r d}\right|^{2} \mathrm{P}_{b}^{t a r}}{a_{1}\left(\left|h_{s r}\right|^{2}+\left|h_{r d}\right|^{2}\right.}\right)\right)}{\left|h_{r d}\right|^{2}}\right] .
$$

We can see $P_{T, \text { min }}$ is a function of the modulation rate $\theta$, the circuit power $P_{C}$ is also the function of the modulation rate $\theta$, then the total power can represent as $P_{t o t}(\theta)$.

$P_{\text {tot }}(\theta)=P_{T, \min } / \xi+P_{C}$.

Thus, the energy efficiency function can express as 


$$
\eta_{E E}=\frac{R_{t o t}(\theta)}{P_{t o t}(\theta)}=\frac{\theta B}{\frac{\left(a_{4}-2^{a_{3} \theta}\right) \sigma_{n}^{2}}{a_{2} \cdot \xi} \cdot\left[\frac{\ln \left(\frac{\left|h_{s r}\right|^{2} \mathrm{P}_{b}^{t a r}}{a_{1}\left(\left|h_{s r}\right|^{2}+\left|h_{r d}\right|^{2}\right)}\right)}{\left|h_{s r}\right|^{2}}+\frac{\ln \left(\frac{\left|h_{r d}\right|^{2} \mathrm{P}_{b}^{\text {tar }}}{a_{1}\left(\left|h_{s r}\right|^{2}+\left|h_{r d}\right|^{2}\right)}\right)}{\left|h_{r d}\right|^{2}}\right]+(\alpha+\beta \theta B)} \cdot
$$

\section{Theoretical Analysis}

Theorem 1. The energy efficiency function $\eta_{E E}$ is a continuous quasi-concave function of $\theta$.

Proof:

According to [8], $\eta_{E E}$ is strictly quasi-concave of $\theta \geq 0$, if the set $C_{\alpha}=\left\{\theta \mid \eta_{E E} \geq \alpha\right\}$ is a strictly convex set for any real value $\alpha$.

Obviously, $\forall \theta>0$, if $\alpha \leq 0, C_{\alpha}=\{\theta \mid f(\alpha, \theta) \geq 0\}$ is a strictly concave set. If $\alpha>0$, the set is $C_{\alpha}=\left\{\theta \mid f(\alpha, \theta)=R(\theta)-\alpha P_{\text {tot }}(\theta) \geq 0\right\}$. As $R(\theta)$ is linear with $\theta, R(\theta)$ is concave on $\theta$, and $P_{\text {tot }}(\theta)$ is also concave on $\theta$, then $f(\alpha, \theta)$ is strict concave with respect to $\theta$.

Suppose $\theta_{1}$ and $\theta_{2}$ are any two points on the contour of $C_{\alpha}$, since $f(\alpha, \theta)$ is strict concave with respect to $\theta, \forall \theta \in\left(\theta_{1}, \theta_{2}\right), f(\alpha, \theta)>\min \left\{f\left(\alpha, \theta_{1}\right), f\left(\alpha, \theta_{2}\right)\right\} \geq 0$ holds, meaning that any $\theta$ between any two points on the contour of $C_{\alpha}$ must lie in the interior of $C_{\alpha}$. Therefore, $\eta_{E E}$ is strictly quasi-convex of $\theta$.

Theorem 2. For $\eta_{E E}$ is a continuous quasi-concave function of $\theta$, the following properties are satisfied:

1) There exists a unique $\theta^{*}$, making $\eta_{E E}^{\prime}(\theta)=0$ must hold.

2) $\forall \theta \in\left(0, \theta^{*}\right], \eta_{E E}^{\prime}(\theta)>0, \eta_{E E}(\theta)$ is strictly increasing.

3) $\forall \theta \in\left[\theta^{*},+\infty\right), \quad \eta_{E E}^{\prime}(\theta)<0, \quad \eta_{E E}(\theta)$ is strictly decreasing.

Proof: See Appendix

\section{Simulation Results}

To corroborate the theoretical analysis, simulations results will be shown in this section. The scenario considered is shown in fig. 2. To make it easier, we put the relay in the straight line between nodes $\mathrm{S}$ and $\mathrm{D}$. The distance between nodes $\mathrm{S}$ and $\mathrm{R}$ is $d_{s d}=1000 \mathrm{~m}$, and between node $\mathrm{S}$ and relay $\mathrm{R}$ is $d_{s r}$. We can get different curves of $P_{T, \min }(\theta)$ and energy efficiency $\eta_{E E}(\theta)$ by adjusting the positions of relay nodes. The system parameters are set as follows: $\eta=0.4$, $B=10 \mathrm{KHz}, \sigma^{2}=-140 \mathrm{dBm} / \mathrm{Hz}$, which are in agreement with the practical values as used in Ref. [9].

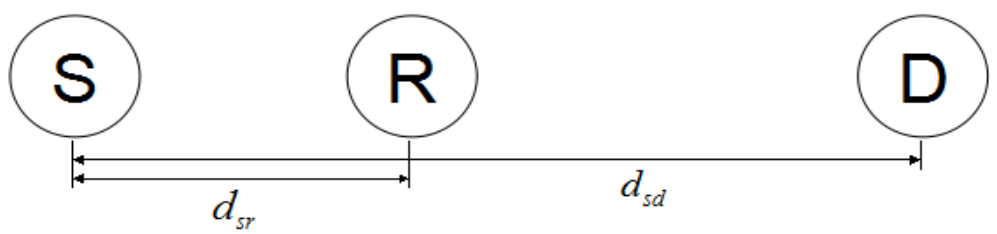

Fig. 2. The scenario considered in the simulation

The channel power gain is evaluated according to $\left|h_{s r}\right|^{2}=G_{0} d_{s r}{ }^{-4},\left|h_{r d}\right|^{2}=G_{0}\left(d_{s d}-d_{s r}\right)^{-4}$, where $G_{0}=-70 \mathrm{~dB}$ as used in [9]. 


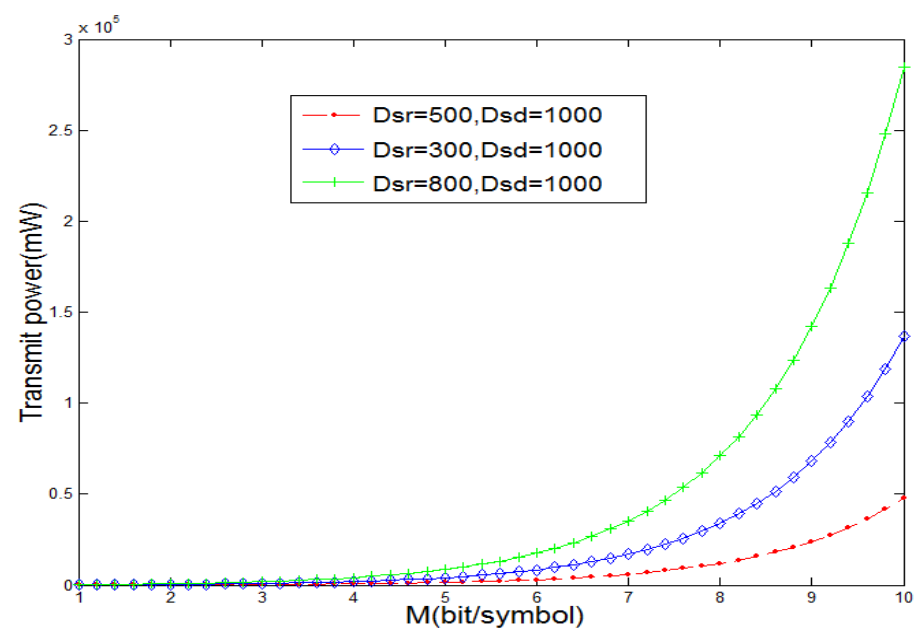

Fig. 3. The minimized transmission power of different relay location

The minimized transmission power with respect to $\theta$ is shown in fig. 3, which satisfied the given BER. We can see that the transmission power is increased with the increase of the number of bits carried by one symbol. When the relay node is in the middle of the node $S$ and $D$, the transmission power is much less than other situation, e.g. node $\mathrm{R}$ is close to node $\mathrm{S}$ or $\mathrm{D}$. By the way, we can also see the concavity of $P_{T}(\theta)$ in this figure intuitively.

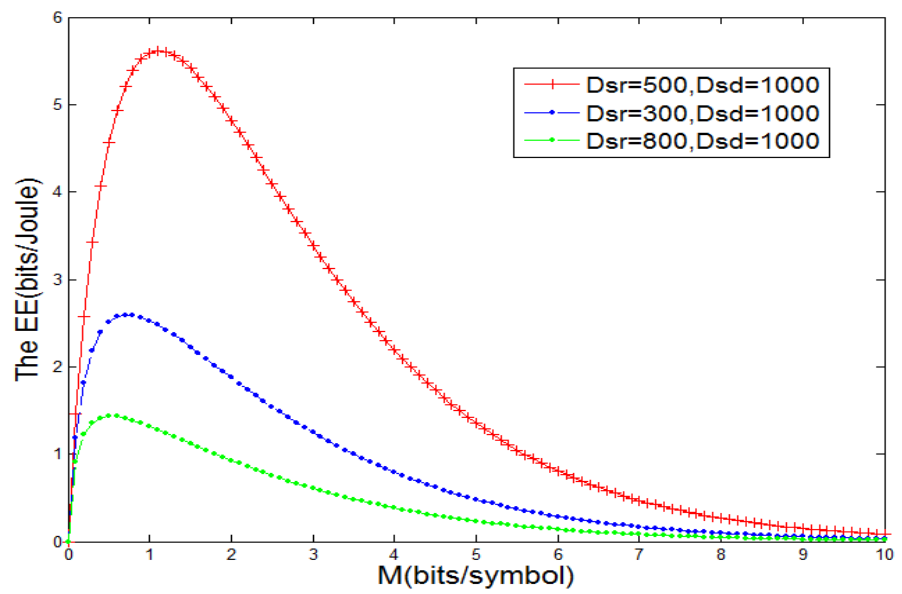

Fig. 4. The trade-off between EE and modulation rate of different relay location

We can find that when the modulation rate increases, the energy efficiency will be first increased and then decreased in fig. 4, but it will continue to drop after the rate develops to a certain degree. We can explain this phenomenon as when we transmit data bits at a high rate, it will consume more energy and the energy efficiency will be low. This meaning that we should trade-off energy efficiency and modulation rate in some practical matters. Moreover, from fig. 4, we can see the EE is better, when the relay node is in the middle of the node S and D. So when we design networks, we should try to put relays in the center of the links.

\section{Conclusions}

In this paper, we have considered a two-hop DF relay system with MQAM modulation. The minimum power consumption is first deduced and we consider the circuit power consumption at the same time. The expression of energy efficiency based on the modulation rate is derived and analyzed, at the mean time, we guarantee that demodulation error rate at receiver is lower than the given value. The mathematical theory analysis shows that the performance of energy efficiency is the quasi-concave function of modulation rate, and when the modulation rate increases, the energy efficiency first increases and then decreases. Finally, the theoretical analysis has been confirmed by simulation results. 


\section{Appendix}

A. Solve the power optimization problem with KKT conditions.

Rewrite the power optimization problem (10) as

$\min P_{T}$

$$
\begin{aligned}
\text { s.t. } & \mathrm{P}_{b}^{S R}+\mathrm{P}_{b}^{R D} \leq \mathrm{P}_{b}^{t a r} \\
& P_{S}, P_{R}>0 .
\end{aligned}
$$

From $P_{T}=P_{S}+P_{R}$ and (8), the above optimization problem can be equivalent to

$$
\begin{array}{ll}
\min & \frac{\left(a_{4}-2^{a_{3} \theta}\right) \sigma_{n}^{2}}{a_{2}\left|h_{s r}\right|^{2}} \cdot \ln \left(\frac{\mathrm{P}_{b}^{S R}}{a_{1}}\right)+\frac{\left(a_{4}-2^{a_{3} \theta}\right) \sigma_{n}^{2}}{a_{2}\left|h_{r d}\right|^{2}} \cdot \ln \left(\frac{\mathrm{P}_{b}^{R D}}{a_{1}}\right) \\
\text { s.t. } & \mathrm{P}_{b}^{S R}+\mathrm{P}_{b}^{R D} \leq \mathrm{P}_{b}^{t a r} \\
& P_{S}, P_{R}>0 .
\end{array}
$$

By using KKT conditions, let $\mathrm{P}_{b}^{S R}=x, \mathrm{P}_{b}^{R D}=y, \frac{\left(a_{4}-2^{a_{3} \theta}\right) \sigma_{n}^{2}}{a_{2}\left|h_{s r}\right|^{2}}=A, \frac{\left(a_{4}-2^{a_{3} \theta}\right) \sigma_{n}^{2}}{a_{2}\left|h_{r d}\right|^{2}}=B$, we can obtain

$$
L\left(x, y, \lambda_{1}, \lambda_{2}, \lambda_{3}\right)=A \ln \left(\frac{x}{a_{1}}\right)+B \ln \left(\frac{y}{a_{1}}\right)-\lambda_{1}\left(\mathrm{P}_{b}^{t a r}-x-y\right)-\lambda_{2} A \ln \left(\frac{x}{a_{1}}\right)-\lambda_{3} B \ln \left(\frac{y}{a_{1}}\right)
$$

The extremum conditions can be expressed as

$$
\left\{\begin{array}{l}
\frac{A}{x}+\lambda_{1}-\lambda_{2} \frac{A}{x}=0 \\
\frac{B}{y}+\lambda_{1}-\lambda_{3} \frac{B}{y}=0 \\
\lambda_{1}\left(\mathrm{P}_{b}^{t a r}-x-y\right)=0 \\
\lambda_{2} A \ln \left(\frac{x}{a_{1}}\right)=0 \\
\lambda_{3} B \ln \left(\frac{y}{a_{1}}\right)=0 \\
\lambda_{i} \geq 0 \quad(i=1,2,3)
\end{array}\right.
$$

From (A-4), we know that $\lambda_{2}=\lambda_{3}=0, \mathrm{P}_{b}^{\text {tar }}-x-y=0$, comprehensive the above conditions, we can obtain that

$$
x=\frac{A}{A+B} \mathrm{P}_{b}^{t a r}, y=\frac{B}{A+B} \mathrm{P}_{b}^{t a r} .
$$

Namely,

$$
\mathrm{P}_{b}^{S R}=\frac{\left|h_{s r}\right|^{2} \mathrm{P}_{b}^{t a r}}{\left|h_{s r}\right|^{2}+\left|h_{r d}\right|^{2}}, \quad \mathrm{P}_{b}^{R D}=\frac{\left|h_{r d}\right|^{2} \mathrm{P}_{b}^{t a r}}{\left|h_{s r}\right|^{2}+\left|h_{r d}\right|^{2}} .
$$

\section{B. Proof of Theorem 2}

According to the strict quasi-concavity of $\eta_{E E}(\theta), \forall \theta \in\left(\theta_{1}, \theta_{2}\right), \min \left\{\eta_{E E}\left(\theta_{1}\right), \eta_{E E}\left(\theta_{2}\right)\right\}<\eta_{E E}(\theta)$ holds [10]. Suppose there exist $\theta_{1}$ and $\theta_{2}$ satisfying $\theta_{1}<\theta_{2}$ and $\eta_{E E}\left(\theta_{1}\right)=\eta_{E E}\left(\theta_{2}\right)=\eta *$. Then $\forall \theta \in\left(\theta_{1}, \theta_{2}\right), \quad \eta^{*}=\min \left\{\eta_{E E}\left(\theta_{1}\right), \eta_{E E}\left(\theta_{2}\right)\right\}<\eta_{E E}(\theta)$, this is contrary to $\eta^{*} \geq \eta_{E E}(\theta)$. Therefore, unique $\theta *$ exists. Moreover, as it says in [8], $\theta *$ must satisfy $\forall \theta \geq 0, \eta_{E E}^{\prime}\left(\theta^{*}\right)\left(\theta-\theta^{*}\right) \geq 0$, thus 
$\eta_{E E}^{\prime}\left(\theta^{*}\right)=0$ must hold. The first claim is proved.

For any $\theta_{1}$ and $\theta_{2}$ satisfying $0<\theta_{1}<\theta_{2} \leq \theta^{*}, \quad \eta_{E E}\left(\theta_{1}\right)=\max \left\{\eta_{E E}\left(\theta_{1}\right), \eta_{E E}\left(\theta^{*}\right)\right\}<\eta_{E E}\left(\theta_{2}\right)$ holds. This meas that $\eta_{E E}(\theta)$ is strictly increasing with $\theta \in\left(0, \theta^{*}\right)$, therefore, $\forall \theta \in\left(0, \theta^{*}\right), \eta_{E E}^{\prime}(\theta)>0$ holds.

The same as above, $\forall \theta_{1}, \theta_{2}$ satisfies $\theta_{1}>\theta_{2} \geq \theta^{*}, \eta_{E E}\left(\theta_{1}\right)=\max \left\{\eta_{E E}\left(\theta_{1}\right), \eta_{E E}\left(\theta^{*}\right)\right\}<\eta_{E E}\left(\theta_{2}\right)$. This means that $\eta_{E E}(\theta)$ is strictly decreasing with $\theta \in\left(\theta^{*},+\infty\right)$, therefore, $\forall \theta \in\left(\theta^{*},+\infty\right)$, $\eta_{E E}^{\prime}(\theta)>0$ holds.

\section{Acknowledgements}

This work is supported by NSF China(61401266, 61571279), Innovation Program of Shanghai Municipal Education Commission (14ZZ096), SRFDP (20133108120015), The Program for Professor of Special Appointment (Eastern Scholar) at Shanghai Institutions of Higher Learning.

\section{References}

[1] Shaoqing W, Jingnan N. Energy efficiency optimization of cooperative communication in wireless sensor networks[J]. EURASIP Journal on Wireless Communications and Networking, 2010.

[2] Li G Y, Xu Z, Xiong C. Energy-efficient wireless communications: tutorial, survey, and open issues[J]. Wireless Communications, IEEE, 2011, 18:28 - 35.

[3] C. Sun and Chenyang Yang, Energy efficiency comparison among direct, one-way and two-way relay transmission Communications (ICC), 2012 IEEE International Conference, 2012 , Page(s): $4288-4293$

[4] Yu H, Li Y, Zhong X, et al. The analysis of the energy efficiency for the decode-and-forward two-way relay networks[C]//Wireless Communications and Networking Conference (WCNC), 2013 IEEE. IEEE, 2013: 2823-2827.

[5] M. Wu, D. Wubben, and A. Dekorsy, "Ber-based power allocation for amplify-and-forward and decode-and-forward relaying systems," in Smart Antennas (WSA), 2011 International ITG Workshop on. IEEE, 2011, pp.1-8.

[6] S. T. Chung and A. J. Goldsmith, "Degrees of freedom in adaptive modulation: a unified view," Communications, IEEE Transactions on,vol. 49, no. 9, pp. 1561-1571, 2001.

[7] C. Isheden and G. P. Fettweis, "Energy-efficient multi-carrier link adaptation with sum rate-dependent circuit power," inProc. IEEE Global Telecommun. Conf., Dec. 2010.

[8] S. Boyd and L. Vandenberghe, Convex optimization.Cambridge Uni-versity Press, 2004.

[9] S. Cui, A. Goldsmith, and A. Bahai, "Energy-constrained modulation optimization," IEEE Trans. Wirel. Commun., vol. 4, no. 5, pp. 2349-360, Sep. 2005.

[10] T. Wang and L. Vandendorpe, On the optimum energy efficiency for flat-fading channels with rate-dependent circuit power, IEEE Transactions on Communications, vol. 61, no. 12, pp. 4910-4921. Dec. 2013 